\title{
A Perspective on Medical Applications of High Temperature Superconductors
}

\section{Samir M Iqbal ${ }^{1^{\star}}$ and Sajid Saleem ${ }^{2}$}

${ }^{1}$ Nano-Bio Lab, Department of Electrical Engineering, Department of Bioengineering, Nanotechnology Research Center, University of Texas at Arlington, Arlington, USA ${ }^{2}$ Department of Electrical and Power Engineering, National University of Sciences and Technology, Karachi, Pakistan

"Corresponding author: Samir M Iqbal, Nano-Bio Lab, Department of Electrical Engineering, Department of Bioengineering, Nanotechnology Research Center, University of Texas at Arlington, Arlington, USA, E-mail: smiqbal@uta.edu

Rec date: Apr 26, 2014, Acc date: Apr 27, 2014, Pub date: May 05, 2014

Copyright: () 2014 lqbal SM, et al. This is an open-access article distributed under the terms of the Creative Commons Attribution License, which permits unrestricted use, distribution, and reproduction in any medium, provided the original author and source are credited.

\section{Editorial}

Superconductivity was first discovered in mercury in 1911 by Dutch physicist Kamerlingh Onnes of Leiden University. Superconductivity has also been discovered in a number of other metallic elements. Collectively these elements are called low temperate superconductors (LTS). In 1986, Alex Muller and Georg Bednorz discovered superconductivity in a brittle ceramic $\mathrm{Ba}-\mathrm{La}-\mathrm{Cu}-\mathrm{O}$ compound at $30^{\circ} \mathrm{K}$ [1]. A number of other compounds like yttrium barium copper oxide (YBCO), bismuth strontium calcium copper oxide (BSCCO) and thallium barium calcium copper oxide (TBCCO) have been found to superconduct at even higher temperatures. The highest superconducting temperature of $138 \mathrm{~K}$ was observed in a thalliumdoped, mercuric cuprate in 1994. A new family of arsenic based superconducting materials termed oxypnictides has been discovered recently. These compounds are collectively called high temperature superconductors (HTS).

Two major requirements for a material to be considered as superconductor are, (i) zero resistance below a certain temperature and (ii) the ability to expel a magnetic field from inside. The temperature at which the resistance of the material goes to zero and perfect conductivity appears is termed the critical temperature, TC. Superconducting materials have to be cooled below their TC using cryogenic liquids such as helium for LTS and nitrogen for HTS. The second fundamental property of a superconductor is its ability to expel a magnetic field from its bulk at a temperature lower than TC. The maximum amount of magnetic field, which can be expelled, is termed as HC. This effect in the superconductor is termed the Meissner effect after the name of its discoverer [2].

Since its discovery, superconductivity has found numerous applications in very diverse fields. Applications of superconductivity can be found in transportation (Maglev), marine and military (propulsion motors, degaussing systems and EMP weapons), particle research (large hadron collider, proton-antiproton collider and electron-proton collider etc., power generation and distribution (fault current limiters, superconducting wires, superconducting magnetic energy storage systems and superconducting transformers etc., information technology \& computing (quantum computers, quantum cryptography and high performance computers etc), electronics \& telecommunications (Superconducting Quantum Interference Device (SQUID) and cellular filters etc.) and medical diagnostic systems.

Medical applications of superconductivity can be divided in to two major categories. First based on superconducting magnets, e.g. MRI and particle accelerators, and second based on the detection of biomagnetism such as SQUID-based magnetoencephalography (MEG) and magnetocardiography (MCG) [3] At present, almost all commercial medical systems use LTS and majority uses NbTi wires or
SQUIDS made of LTS material. Use of LTS materials means we cannot use a cheap cryogen like nitrogen. Liquid helium or complex cryogenic systems are required to cool LTS below its TC. This requirement adds extra complexity and cost to the overall system.

Obvious solution to circumvent this problem is to use materials with higher TC and HC. Liquid nitrogen and simpler cryogenic solutions can be then employed. However HTS have their own associated problems, which need addressing to make them viable for widespread applications. Currently HTS based medical systems are either in research laboratories or have very limited experimental/ commercial setups. Major reason being that applications at $77 \mathrm{~K}$ (for HTS) are much more challenging than at $4.2 \mathrm{~K}$ (for LTS), regardless of the values of TC and HC [4,5] The future of HTS medical applications is a persistent question and can only be answered once the scientific and technological challenges hindering the large-scale application are understood.

First, superconductivity is inherently complex as it is a multidisciplinary field encompassing as diverse areas as thermodynamics, quantum physics, cryogenics, material engineering, electrical and electronic engineering. One parameter to gauge the complexity of any field is the time gap between the actual discovery and its practical application. Superconductivity beats many other fields in terms of this measure e.g. transistor development and optical communication. Time gap between initial transistor development and first functional IC was only 11 years. Communication by light was realized in 1880 by Alexander Graham Bell, 22 years after its first concept in 1858 by J. Tyndall. However in case of superconductivity, it took 50 years after its discovery in 1911 to make the first NbTi magnet. Twenty six years passed between the Nb3Sn magnet discovery and in the production of first ever industrial Nb3Sn magnet. HTS materials were discovered in 1987 and the market penetration has started only now, 25 years later [3]. Similarly, we have yet to see any practical applications of oxypnictides since their discovery in 2008 due to their complex nature.

The second challenge has to deal with the material properties that result in high values of TC and HC. These properties include high normal state resistance, proximity of superconductivity to competing Anti-Ferromagnetic (AF) state, large anisotropic ratios and sensitivity of superconducting properties to local nonstoichiometry [5]. These lead to the lack of widespread applications of HTS. In addition, absence of any validated microscopic theory of superconductivity in HTS materials means we cannot predict new superconducting materials with higher TC values.

The third challenge is the geometry. The geometrical shapes of HTS wire are limited. It is mostly available in tape format. Fabrication of HTS wire (tape) requires specialist technology and process machinery due to this limitation. However, recently discovered FeAs-122 
Citation: Iqbal, Saleem (2014) A Perspective on Medical Applications of High Temperature Superconductors. J Bioeng Biomed Sci 4: e119. doi:

Page 2 of 2

belonging to the large family of Fe pnictides, has the potential to be formed as a round wire due to its lower anisotropy value compared to other HTS materials. The flip side is that FeAs-122 has lower TC values than other HTS compounds (up to $38^{\circ} \mathrm{K}$ ). Wire length of HTS is also limited due to its less manipulability than NbTi. Metallurgical difficulties in preparing high quality HTS wires and tapes in different geometries limit the use of HTS based medical systems with higher TC values.

The most important factor hindering the applications of HTS medical systems is the overall cost. Even if all of the issues related to HTS application were addressed, the bottom line would always be the economics. If we can use HTS materials using nitrogen as cryogenics, would the cost reduction justify the use of HTS? HTS wires and materials are at present too expensive to compete with $\mathrm{NbTi}$ wire and other LTS materials. As a comparison, $\mathrm{NbTi}$ wire is easily available in different forms at a cost of around \$2/kA-m compared to almost $100 \times$ higher price of HTS wire and hence rules out the use of HTS wire.

We believe that widespread medical use of HTS materials will occur only with the advent of a new generation materials based on non- cryogenic sensors or with the discovery of room temperature superconductivity. This would ensure that HTS materials are less expensive, more compact, practically maintenance and cryogenic.

\section{References}

1. Bednorz JG, Müller KA (1986) Possible highT c superconductivity in the Ba-La-Cu-O system. Zeitschrift für Physik B 64: 189-193.

2. Takahashi H, Igawa K, Arii K, Kamihara Y, Hirano M, et al. (2008) Superconductivity at $43 \mathrm{~K}$ in an iron-based layered compound LaO1xFxFeAs. Nature 453: 376-378.

3. Flükiger R (2012) Overview of Superconductivity and Challenges in Applications. Rev Accl Sci Tech 05: 1-23.

4. Sarrao J, Wai-Kwong K (2006) Basic Research Needs for Superconductivity. Report of the Basic Energy Sciences Workshop on Superconductivity 8-11.

5. Gurevich A (2011) To use or not to use cool superconductors? Nature Materials 10: 255-259. 